\title{
Evidências científicas acerca das atividades biológicas de uma planta nativa do Nordeste brasileiro - o pião roxo
}

\section{Scientific evidence on the biological activities of a native plant in the Brazilian Northeast - the purple top}

\author{
Maria Lysete de Assis Bastos'
}

\section{RESUMO}

Objetivo:identificar evidências científicas acerca das atividades biológicas da J. gossypiifolia (pião roxo).Métodos:revisão integrativa nas bases de dados Literatura Latino-Americana e do Caribe em Ciências da Saúde,Medical Literature Analysis and Retrieval System Online, SCOPUS, Biblioteca Virtual de Saúde e Scientific Electronic Library Online.Foram selecionados 16 artigos, após a aplicação dos critérios de inclusão e exclusão. Resultados:a maioria dos estudosé de origem nacional, publicadoem português e oriundo do nordeste. Quanto ao período de publicação, mais de $30 \%$ foram publicados em 2006. Nas atividades biológicas, referem-se àtóxica (43,7\%), cicatrizante (3I,2\%), anti-inflamatória ( $12,5 \%)$, cicatrizante/anti-inflamatória, cicatrizante/tóxica e anticoagulante, antioxidante e tóxica (6,2\%) para cada uma, respectivamente. Conclusão:Os estudosassinalam para a continuação de pesquisas referentes ao uso popular do pião roxo, principalmente para tratar feridas, dor e inflamação, visando a promoção da saúde na Atenção Básica, conforme a Política Nacional de Práticas Integrativas e Complementares no SUS.

Descritores: Cicatrização de feridas; Medicina tradicional; Toxicidade.

\begin{abstract}
Objective: to identify the scientific evidence about the biological activities of J. gossypiifolia (purple top). Methods: Integrative review in Latin American and Caribbean Literature in Health Sciences, Medical Literature Analysis and Retrieval System Online, SCOPUS, Virtual Health Library and Scientific Electronic Library Online. We selected 16 articles, after applying the inclusion and exclusion criteria. Results: most of the studies are of national origin, published in Portuguese and coming from the northeast of Brazil.As for the publication period, more than $30 \%$ were published in 2006. In biological activities, they refer to the toxic (43.7\%), healing (3I.2\%), anti-inflammatory ( $12.5 \%)$, healing / anti-inflammatory, healing / toxic and anticoagulant, antioxidant and toxic (6.2\%) for each, respectively. Conclusion: The studies indicate the continuation of researches related to the popular use of the purple top, mainly to treat wounds, pain and inflammation, aiming at health promotion in Primary Care, according to the National Policy of Integrative and Complementary Practices in SUS.
\end{abstract}

Descriptors: Wound Healing; Medicine Traditional; Toxicity. 


\section{INTRODUÇÃO}

O aproveitamento de recursos naturais tem apresentado uma tendência cada vez mais crescente na saúde, em virtude de vantagens econômicas, menor possibilidade deefeitoscolaterais ao organismo e maior acesso à população,ganhando relevanteespaço de modo complementar às terapias medicamentosas alopáticas.

Há relatos da utilização das plantas como alternativa de tratamento para recuperação da saúde que coincidem com a existência da espécie humana, datando até 3000 anos $\mathrm{aC}^{(1)}$. Desta forma, o homem adquiriu conhecimentos empíricos que foram repassados às demais gerações, demonstrando notável evolução no emprego deste recurso natural ao longo dos anos, desde as formas mais simples de tratamento local, até formas sofisticadas de fabricação industrial utilizadas pelo homem moderno(2).

Tendo em vista que uma enorme parcela da população vive constantemente cercada por condições precárias, e que em muitos locais do Brasil, os cuidados à saúde e sanitários são inacessíveis, presume-se que as consequências dessas condições favorecem a população ou a comunidade para a busca de plantas medicinais pela sua facilidade de aquisição.

Devido a isso, a ciência vem demonstrando que as plantas medicinais têm grande influência na manutenção das condições de saúde das pessoas. Isto se deve ao aumento de estudos com as mesmas, o que levam à comprovação científica da ação terapêutica de várias espécies vegetais conhecidas popularmente, mas, sobretudo, ao fato de que o tratamento com as citadas plantas é parte da cultura popular, sendo utilizada e difundida por muitas gerações ${ }^{(3)}$.

A descoberta de compostos bioativos é oriunda, principalmente,dos diversosestudos químicosrealizados a partir deplantas medicinais e suasaplicaçõesvêm sendo difundidaspara o desenvolvimento de novos fármacos ${ }^{(1)}$. Estima-se que existam de 25.000 a 75.000 espécies vegetais utilizadas na medicina tradicional nomundo. No entanto, apenas $1 \%$ foi validada por estudos científicos e investigadas sob seu aspecto fitoquímico ${ }^{(4)}$, o que justifica o crescente interesse de profissionais da área, no estudo de processos de isolamento e no desenvolvimento de métodos para síntese de tais compostos.

É nesse contexto que se evidencia o papel do enfermeiro na busca por novas opções terapêuticas, por meio da utilização das plantas medicinais como parte inerente do exercício de cuidar, fortalecendo a autonomia e a integralidade das ações em sua prática assistencial, uma vez que o universo da saúde transcende os muros das instituições hospitalares e até mesmo da rede básica ${ }^{(5)}$.

Com o intuito de normatizar e harmonizar o exercício da medicina tradicional na população brasileira e atender à recomendação da Organização Mundial da
Saúde (OMS) de incentivar tal prática, o Brasil, em 2005, elaborou a Política Nacional de Práticas Integrativas e Complementares (PNPIC), trazendo as diretrizes e ações para inserção de serviços e produtos relacionados à medicina tradicional chinesa - acupuntura, homeopatia e plantas medicinais e fitoterapia no âmbito do Sistema Único de Saúde (SUS) ${ }^{(6)}$.

Nessa perspectiva, estudos baseados nas propriedades biológicas das plantas medicinais estão sendo desenvolvidos, dentre eles, encontram-se as pesquisas com o uso de Jatropha gossypiifolia L.,espécie amplamente distribuída nos países de clima tropical e nas regiões semiáridas da África e das Américas, a qual está inclusa na Relação Nacional de Plantas Medicinais de Interesse ao SUS (RENISUS) ${ }^{(7)}$.

A J. gossypiifolia, conhecida comopião roxo ou pinhão -roxo, pertence à família Euphorbiaceae; gênero Jatropha; espécie gossypiifolia,apresenta-se como uma árvore de foIhas alternas grandes, flores roxas, frutos pequenos e capsulares.Esta espécie vegetal se destaca pelo seu potencial analgésico e anti-inflamatório, assim como por ser utilizada para o tratamento de doenças da pele, cicatrização de feridas, disenteria, lepra, artrite, otite, alopecia, doenças venéreas, dores de estômago, obstruções do trato abdominal, reumatismo e mordida de animais venenosos, tornando-se pertinente na prática da medicina popular baseada nos conhecimentos específicos desta planta ${ }^{(8)}$.

Entretanto, a J. gossypiifolia tem sido relatada pelo senso comum, como uma das principais plantas tóxicas conhecidas no Brasil( ${ }^{(9)}$. Esta informação indica para a necessidade de um levantamento da produção de estudos relacionados àreferida espécie vegetal. Desse modo, a pesquisa pode contribuir como fonte de conhecimento para o desenvolvimento de estudos experimentais, auxiliando na complexa avaliação da relação risco/benefício da utilização desta planta.Assim, o objetivo foi identificar as evidências científicas acerca das atividades biológicas da J. gossypiifolia(pião roxo).

\section{MÉTODOS}

Trata-se de uma revisão integrativa da literatura, que se configura como uma modalidade de pesquisa que visa reunir e sintetizar os estudos realizados sobre determinada temática para, a partir disto, construir uma conclusão, baseando-se nos resultados evidenciados em cada estudo ${ }^{(10)}$. Representa, ainda,mais um recurso para a construção do conhecimento em saúde e dado a sua natureza, pode subsidiar o desenvolvimento e a acurácia da prática clínica e consequente intervenções que tenham como resultados a segurança do paciente.

Para desenvolvimento da revisão integrativa foram percorridas seis etapas: I) estabelecimento dapergunta norteadora; 2) amostragem ou busca na literatura; 3) ca- 
tegorização dos estudos; 4) avaliação dos estudos incluídos na revisão; 5) interpretação dos resultados; 6) síntese do conhecimento ou apresentação da revisão,a fim de garantir o rigor metodológico do estudo e garantir que a questão norteadora seja respondida ${ }^{(10)}$.Desse modo, realizou-se a seguinte pergunta norteadora: quais atividades biológicas apresenta a espécie vegetal J. gossypiifolia?

A coleta dos dados ocorreu entre janeiro e abril de 2016, por meio do cruzamento da literatura das bases de dados: Literatura Latino-Americana e do Caribe em Ciências da Saúde(LILACS),Medical Literature Analysis and Retrieval System Online (MEDLINE), SCOPUS, Biblioteca Virtual de Saúde(BVS)e Scientific Electronic Library Online(Scielo).

Utilizaram-se como critérios de inclusão, publicações nacionais e internacionais divulgadas em língua portuguesa, inglesa e espanhola dos últimos 10 anos, publicados entre o período de 2006 a 2016, disponíveis online na íntegra e gratuitamente nas bases de dados, que apresentassem em sua discussão considerações sobre as atividades biológicas da Jatropha gossypiifolia. Optou-se por excluir estudos que correspondiam a editoriais, resumos de congressos, anais, opiniões, comentários e artigos repetidos em mais de uma base de dados.

A partir das palavras-chave utilizou-se o descritor primário Jatropha gossypiifolia L.agregado com os seguintes descritores secundários: cicatrização de feridas/wound healing/cicatrización de heridas, toxicidade/toxicity/toxicidad, medicina popular/folk medicine/medicina popular.Como estratégia de busca, a pesquisa foi realizada a partir do cruzamento apenas dos descritores na língua inglesaseparados pelo operador boleano AND.

Ao todo, encontrou-se 152 artigos, dos quais 99 $(65,1 \%)$ foram previamente excluídos por repetição nas bases de dados e 37 (24,3\%) por não se adequarem aos critérios de inclusão. Assim, a amostra obtida para a realização da revisão foi constituída por 16 (10,5\%) estudos.

O conteúdo dos estudos foi analisado pela leitura e releitura de seus resultados, o que permitiu identificar características relevantes que se repetiam ou se destacavam, possibilitando a organização dos dados em categorias por atividade de ação. Considera-se acategorizaçãocomo uma operação de classificação de elementos constitutivos de um conjunto(II).

Após a leitura na íntegra, e com o intuito de fazer uma síntese, elaborou-se um instrumento de coleta, contemplando as informações: título/periódico, tipo de estudo/nível de evidência, país, idioma, ano de publicação, objetivos, atividade biológica e amostra.

Os artigos selecionados foram classificados quanto ao nível de evidência, considerando-se o delineamento utilizado para o desenvolvimento do estudo, empregando-se o sistema de classificação composto por sete níveis: nível I - evidências oriundas de revisões sistemáticas ou meta-análise de relevantes ensaios clínicos; nível II evidências derivadas de pelo menos um ensaio clínico randomizado controlado bem delineado; nível III - ensaios clínicos bem delineados sem randomização; nível IV - estudos de coorte e de caso-controle bem delineados; nível $V$ - revisão sistemática de estudos descritivos e qualitativos; nívelVI - evidências derivadas de um único estudo descritivo ou qualitativo e nível VII - opinião de autoridades ou relatório de comitês de especialistas. As evidências pertencentes aos níveis I e II são consideradas fortes, de III a V evidências moderadas e VI e VII evidências fracas ${ }^{(12)}$.

Quanto às atividades biológicas da J. gossypiifolia L., estas foram alocadas em seis categorias temáticas. Assim, para o processamento da análise, organizaram-se os resultados em dois quadros. No primeiro mostra-se a caracterização dos estudos, contendo neste item os dados referentes ao título/periódico, tipo de estudo/nível de evidência, país e ano de publicação. Já os objetivos, atividades biológicas e amostra (n) estão dispostos no segundo quadro. Para melhor compreensão, a atividade biológica foi distribuída nas categorias temáticas: I) atividade cicatrizante, 2) tóxica, 3) anti-inflamatória, 4) cicatrizante/anti-inflamatória, 5) cicatrizante/tóxica e 6) anticoagulante, antioxidante e tóxica.

\section{RESULTADOS}

\section{a) Caracterização dos estudos}

De acordo com o Quadro I, a maioria (62,5\%)foi publicada na língua portuguesa e $6(37,5 \%)$ na inglesa.A despeito disso, apenas um estudo $(6,3 \%)$ foi indiano e 15 $(93,7 \%)$ de origem brasileira, sendo seis oriundas do estado do Maranhão, seguido por quatro do Rio Grande do Norte, três da Paraíba, um do Piauí e outro de Pernambuco. Quanto ao período de publicação dos artigos, cinco $(31,2 \%)$ foram publicados em 2006, e a mais recente e única publicação em 2016 representa 6,2\% da amostra. Os artigos estão expostos por ordem alfabética

Quanto ao tipo de estudo/nível de evidência correspondente (quadro I) observa-se que I $3(81,2 \%)$ dos artigos são experimentais com nível de evidência $\mathrm{VI}$ e três (I8,7\%) são de revisão com nível de evidência $V$.

\section{b) Categorias temáticas, segundo atividade biológica}

O detalhamento dos objetivos dos estudos selecionados está apresentado no quadro 2. Nele, também se observam que sete $(43,7 \%)$ das pesquisas referem-se à categoria temática, atividade tóxica, cinco $(31,2 \%)$ à cicatrizante, duas ( $12,5 \%)$ à anti-inflamatória, uma $(6,2 \%)$ à cicatrizante/anti-inflamatória, uma $(6,2 \%)$ cicatrizante/tóxica e uma $(6,2 \%)$ à anticoagulante, antioxidante e tóxica. 
QUADRO 1 - Distribuição dos estudos segundo autor(es), de acordo com título/periódico, tipo de estudo/nível de evidência, local e ano de publicação. Maceió-Al, 2017, n = 16

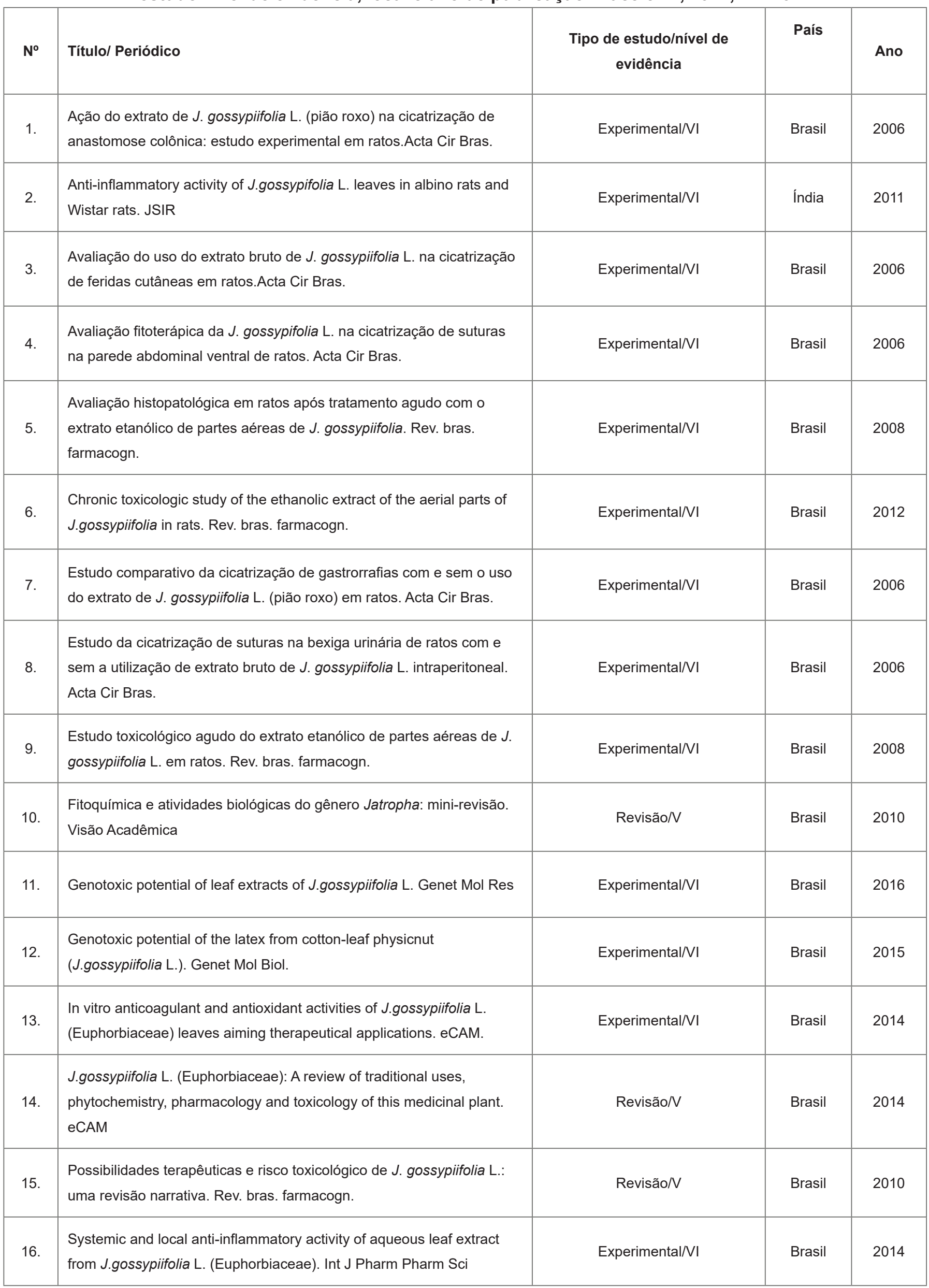




\section{QUADRO 2 - Distribuição dos estudos segundo autor(es), de acordo com objetivo, atividade biológica e} amostra de evidência. Maceió-Al, 2017, $n=16$

\begin{tabular}{|c|c|c|c|}
\hline No. & Objetivo & Atividade biológica & $\begin{array}{c}\text { Amostra } \\
\text { (ratos) }\end{array}$ \\
\hline 1. & $\begin{array}{l}\text { Analisar a ação da J. gossypiifolia L. (pião roxo) na cicatrização de } \\
\text { anastomose colônica em ratos. }\end{array}$ & Cicatrizante e anti-inflamatória & 40 \\
\hline 2. & $\begin{array}{l}\text { Avaliação da atividade antinflamatória local e sistémica de J. gossypiifolia } \\
\text { L. usando modelos animais. }\end{array}$ & Anti-inflamatória & 6 \\
\hline 3. & $\begin{array}{l}\text { Avaliar os aspectos morfológicos do processo cicatricial de feridas } \\
\text { cutâneas abertas de ratos com uso do extrato bruto de J. gossypiifolia L. }\end{array}$ & Cicatrizante & 60 \\
\hline 4. & $\begin{array}{l}\text { Avaliar a influência da administração intraperitoneal da J. gossypiifolia } \\
\text { L., na cicatrização de suturas da parede abdominal ventral de ratos, } \\
\text { observando aspectos macroscópicos, tensiométricos e microscópicos. }\end{array}$ & Cicatrizante & 40 \\
\hline 5. & $\begin{array}{l}\text { Pesquisar as alterações histopatológicas em vísceras de ratos sob } \\
\text { tratamento agudo com o Extrato Etanólico (EE) da J. gossypiifolia L. }\end{array}$ & Tóxica & 24 \\
\hline 6. & $\begin{array}{l}\text { Avaliar a toxicidade crônica do Extrato Etanólico }(E E) \text { de J. gossypiifolia L., } \\
\text { Euphorbiaceae, em ratos, de forma a gerar dados que permitam analisar } \\
\text { a relação risco/benefício de uma eventual utilização terapêutica dessa } \\
\text { espécie. }\end{array}$ & Tóxica & 40 \\
\hline 7. & $\begin{array}{l}\text { Avaliar o uso do extrato bruto de J. gossypiifolia L. na cicatrização de } \\
\text { gastrorrafias em ratos. }\end{array}$ & Cicatrizante & 40 \\
\hline 8. & $\begin{array}{l}\text { Comparar as alterações macroscópicas e histológicas proporcionadas pelo } \\
\text { uso do extrato bruto da J. gossypiifolia L. intra-peritonial, na cicatrização de } \\
\text { suturas realizadas na bexiga urinária de ratos. }\end{array}$ & Cicatrizante & 24 \\
\hline 9. & $\begin{array}{l}\text { Avaliação da toxicidade aguda do Extrato Etanólico (EE) de partes aéreas } \\
\text { de J. gossypiifolia L. }\end{array}$ & Tóxica & ND \\
\hline 10. & $\begin{array}{l}\text { Salientar a importância deste gênero e estimular novas linhas de pesquisa } \\
\text { na investigação de compostos bioativos. }\end{array}$ & Cicatrizante & NA \\
\hline 11. & $\begin{array}{l}\text { Avaliar os efeitos citotóxicos, genotóxicos e efeitos mutagênicos de extratos } \\
\text { etanólico e aquoso de folhas de J. gossypifolia, utilizando o sistema de } \\
\text { teste Allium cepa. }\end{array}$ & Tóxica & NA \\
\hline 12. & $\begin{array}{l}\text { Analisar os efeitos tóxicos, citotóxicos e genotóxicos do látex de } \mathrm{J} \text {. } \\
\text { gossypiifolia por meio do sistema de teste de Allium cepa. }\end{array}$ & Tóxica & NA \\
\hline 13. & $\begin{array}{l}\text { A avaliação da atividade anticoagulante e antioxidante das folhas de } \mathrm{J} \text {. } \\
\text { gossypifolia, buscando novos usos terapêuticos para esta planta. }\end{array}$ & $\begin{array}{l}\text { Anticoagulante, antioxidante e } \\
\text { tóxica }\end{array}$ & ND \\
\hline 14. & $\begin{array}{l}\text { Proporcionar uma atualização no panorama geral das utilizações } \\
\text { tradicionais, bem como a fitoquímica, farmacologia e os dados de } \\
\text { toxicidade da espécie J. gossypifolia L. }\end{array}$ & Tóxica & ND \\
\hline 15. & $\begin{array}{l}\text { Apresentar fonte de informação atualizada aos interessados em } \\
\text { desenvolver pesquisas que ajudem na complexa avaliação da relação } \\
\text { risco/benefício do uso terapêutico de J. gossypiifolia e, consequentemente, } \\
\text { a promoção do uso racional dessa planta (e de derivados) na medicina } \\
\text { popular }\end{array}$ & Cicatrizante e tóxica & 20 \\
\hline 16. & $\begin{array}{l}\text { Avaliar a atividade anti-inflamatória do extrato aquoso da folha de } \mathrm{J} \text {. } \\
\text { gossypifolia e caracterizar seu constituinte fitoquímico. }\end{array}$ & Anti-inflamatória & 24 \\
\hline
\end{tabular}

ND - Não definido; NA - Não se aplica, por se referir a estudo in vitro. 


\section{DISCUSSÃO}

Dos 16 artigos do estudo, a maioria está escrito em português, origem nacional e procede da região nordeste.Estes dados corroboram com estudo, que descreve a J. gossypiifolia como uma planta medicinal conhecida na América Latina e Índia, com vasta distribuição no nordeste brasileiro, pela compatibilidade com temperaturas elevadas. Ela pertence à família Euphorbiaceae, considerada a terceira com maior número de espécies vegetais na caatinga, potencial agrícola na produção de biocombustível, alimento animal e uso medicinal ${ }^{(13)}$.

O bioma caatinga é único pela sua heterogeneidade, com comunidades nativas de baixo poder aquisitivo, que guardam uma vasta farmacopeia popular. Nesse aspecto, identificou-se em um estudo, que as famílias Fabaceae, Anacardiaceae e Euphorbiaceae dessa região exibem grande potencial para compostos bioativos. Pesquisadores do referido estudo sugeriram investigações etnofarmacológicas com estas famílias, na busca por novos fitoterápicos, como estratégia para uso sustentável dos recursos naturais e para impulsionara economia da região, por meio de arranjos produtivos locais (APL) pelo cultivo de plantas medicinais ${ }^{(14)}$.

Dessa forma, pode-se inferir com base na literatura, que por ser o nordeste, uma região ainda muito carente de assistência à saúde e um espaço geográfico em que a J. gossypiifolia prolifera-se facilmente, e que a maioria dos estudos tenham sido realizados por pesquisadores do nordeste do Brasil.

Quanto ao período de publicação dos artigos, o maior percentual (31,2\%) ocorreu em 2006, decrescendo nos anos seguintes, até 2016. Assim, observa-se que há um déficit significativo no quantitativo de produções científicas sobre as atividades biológicas do pião roxo, como é conhecida a J. gossypiifolia, no Brasil. Pode-se inferir que a escassez de publicações sobre o tema seja decorrente da baixa expressividade da caatinga, no setor produtivo nacional.Todavia, é necessário estimular a continuação de estudos para obtenção de suas propriedades biológicas com baixa ou nenhuma toxicidade ${ }^{(15)}$.

AJ. gossypifolia tem sido usada popularmente para tratar diversas afecções, tais como reumatismo, úlceras, hidropsias, hipertensão, cicatrização de feridas, infecções gengivais, dor de estômago, eczema, diarreias, hemorragias, diabetes, câncer e febre ${ }^{(16)}$.Estudo comparativo com e sem uso de extrato desta espécie na cicatrização de gastrorrafias em ratos demonstrou que o extrato contribuiu com o favorecimento do processo cicatricial, aumentando a sua resistência ao teste de resistência à insuflação de ar atmosférico. No terceiro dia de pós-operatório e em sua análise microscópica, observou-se quue favoreceu a cicatrização, aumentando a coaptação das bordas e reduzindo a inflamação aguda no sétimo dia de observação(17).
Outro estudo que avaliou a ação do extrato bruto em feridas cutâneas abertas de ratos evidenciou melhora comparativa na cicatrização das lesões tratadas com os extratos.A propriedade cicatrizante da J.gossypiifoliafoi pesquisada também, por meio da aplicação tópica do extrato bruto em feridas cutâneas de ratos,com resultados positivospara esta atividade biológica ${ }^{(18)}$.

Rotineiramente utiliza-se o rato como modelo experimental do estudo da cicatrização e teste de extratos vegetais e drogas na pele, pelo fato dele já ter sido padronizado por muitas pesquisas, o que corrobora com os achados deste trabalho, visto que nos estudos experimentais que constituíram significativa parte da amostra, o rato é predominantemente o modelo animal mais utilizado, devido principalmente ao pequeno porte, fácil aquisição e padronização no que diz respeito à idade, peso, sexo, alojamento, alimentação, cuidados de limpeza e manipulação experimental|(19).

Estudo experimental com ratos mostrou a ação do extrato da J. gossypiifolia na cicatrização de anastomose colônica, concluindo que houve influência positiva na força da anastomose no terceiro dia pós-cirurgia e ocorrência de pequeno favorecimento no processo cicatricial ${ }^{(20)}$. Entretanto, um estudo que avaliou a cicatrização de suturas na bexiga de ratos não identificou efeito cicatrizante do referido extrato, aplicado por via intraperitoneal (21). Da mesma forma, outro estudo que avaliou a ação fitoterápica desta planta, não evidenciou melhora significativa no processo cicatricial de sutura em parede abdominal de ratos na concentração de $100 \mathrm{mg} / \mathrm{mL}$, sendo administrada a dose de $100 \mathrm{mg} / \mathrm{kg}$ de peso(22).

A cicatrização é um processo biológico complexo que envolve eventos celulares, fisiológicos e bioquímicos,abrangendo fases distintas, tais como inflamação, quimiotaxia, proliferação celular, diferenciação e remodelação, sendo essencial para manter a integridade do organismo ${ }^{(23)}$.

A atividade anti-inflamatória da J. gossypiifolia foi comprovada frente ao edema induzido por TPA (12-Otetradecanoylphorbol-13-acetate) em orelhas de ratos e contra a resposta do edema de pata induzido por carragenina ${ }^{(24)}$. Pesquisa sobre a atividade anti-inflamatória sistêmica e local dos extratos aquosos de J. gossypiifolia descreveram a presença acentuada de flavonoides e compostos fenólicos nesta planta. Tais metabólitos têm diversas atividades farmacológicas, principalmente a anti-inflamatória. A ação dos flavonoides é relevante,pelo fato de serem opção alternativa, para o tratamento de processos inflamatórios ${ }^{(25)}$.

Estudo citotóxico, relacionado ao extrato etanólico desta espécie vegetal, identificou sinais de toxicidade neurológica, gastrointestinal e danos pulmonares, em animais tratados com doses por via oral, confirmando que estes dados são associados principalmente à alta por- 
centagem de letalidade em doses próximas aos níveis terapêuticos indicam toxicidade crônica( ${ }^{(9)}$. Por outro lado, outra pesquisa sobre a toxicidade aguda da J. gossypiifolia demonstrou que o tratamento agudo de ratos Wistar com o extrato etanólico de partes aéreas desta planta promoveu alterações histopatológicas pouco significativas em fígado e pulmão de animais tratados com a maior dose experimental $(5 \mathrm{~g} / \mathrm{kg})$, ratificando que o produto possui toxicidade aguda oral relativamente baixa ${ }^{(26)}$.

Entretanto, foi evidenciada a ausência de citotoxicidade in vitro contra células humanas ao demonstrar uma toxicidade aguda relativamente baixa, provando, ainda, ser uma boa fonte de compostos antioxidantes, sendo essa capacidade sequestradora de radicais livres atribuída à presença de flavonoides ${ }^{(27)}$.

A limitação deste estudo refere-se à baixa ocorrência de pesquisas etnofarmacológicas e pré-clínicas que explorem mais amplamente as atividades biológicas da J. gossypiifolia, no âmbito da medicina popular.

\section{CONCLUSÃO}

As publicações sobre atividades biológicas do pião roxo no tratamento de feridas têm sido tênues, contudo, os resultados apresentados nesta revisão comprovam ampla utilização da espécie vegetal J.gossypiifoliana medicina popular esugerem mostrar potencial para o desenvolvimento de novos fármacos e/ou produtos biotecnológicos. Entretanto, quanto suas atividades biológicas, estudos adicionais com abordagem química, farmacológica e clínica são necessários com esta planta, para melhor elucidar os mecanismos de ação dos seus compostos bioativos. Observou-se, também, que as pesquisas evidenciaram a ação anti-inflamatória do pião roxo, contudo, seus potenciais cicatrizantes e citotóxicos não foram totalmente elucidados, por haver resultados divergentes entre estudos com abordagens semelhantes.

Estes dados trazemsubsídios para continuação de estudos referentes ao uso popular dopião roxo, principalmente, no tratamento de feridas, dor e inflamação, para que possa ser utilizado com segurança para fins de promoção da saúde de usuários na Atenção Básica, conforme a Política Nacional de Práticas Integrativas e Complementares no SUS. 


\section{REFERÊNCIAS}

1. Ribeiro KS, Guimarães ALA. $\bigcirc$ uso de medicamentos à base de plantas medicinais por médicos do SUS no município de Teresópolis/RJ. Rev. Agrogeoambiental. 20 I 3; I:6 I-5. doi: http://dx.doi.org/ |0.18406/23 |6-|8|7v In I20 I358|

2.Holz DT,Vogel-Ely C, Müller NTG, Fasolo D. Conhecimento empírico versus conhecimento científico e análise fitoquímica de espécies medicinais cultivadas por uma associação de Santo Ângelo, Rio Grande do Sul. Revista Biociências. 2013; 19(I): I2- 23. [citado 2016 Mar 6]. Disponível em: http://periodicos.unitau.br/ ojs-2.2/index.php/biociencias/article/viewFile//66I// I83.

3. Nascimento WMC, Melo OF, Silva IF, Souza FL. Plantas medicinais e sua utilização pelas comunidades do município de Sobral, Ceará. Sanare. 20I3; I2(I):46-53. [citado 2017 Ago 24]; Disponível em: https://sanare.emnuvens.com.br/ sanare/article/view/328/262.

4. Bastos RAA, Lopes AMC. A fitoterapia na rede básica de saúde: o olhar da enfermagem. Rev. bras. ciênc. saúde.20I0; I4(2):2I-8.doi: http://dx.doi.org//0.4034/ RBCS.2010.14.02.03

5. Almeida LCT, Tenório LMMC, Verissímo RCSS, Lúcio IML, Bastos MLA. Potencial antimicrobiano do óleo de coco no tratamento de feridas. Rev Rene.2012; I3(4):880-7.doi:http://dx.doi.org// 0.15253/rev\%20rene.v13i4.4053

6. Ministério da Saúde (BR). Política Nacional de Práticas Integrativas e Complementares no SUS - PNPIC Secretaria de Atenção à Saúde. Departamento de Atenção Básica. Brasília: Ministério da Saúde; 2005.

7. Ministério da Saúde (BR). Renisus. Relação nacional de plantas medicinais de interesse ao SUS. Espéciesvegetais. Brasília: Ministério da Saúde; 201 I.

8. Félix-Silva J, Gomes JAS, Barbosa LMQ, Pinheiro ITMG, Soares LAL, Silva-Junior AA et al. Systemic and local anti-inflammatory activity of aqueous leaf extract from Jatropha gossypiifolia L. (Euphorbiaceae). Int J Pharm Pharm Sci. 2014; 6(6): I42-5. [citado 2016 Apr 9]. Disponível em: http://www. ijppsjournal.com/Vol6lssue6/933I.pdf.

9. Mariz SR, Cerqueira GS, Araújo JGD, Ramalho JA, Palomaro TV, Duarte JC et al. Chronic toxicologic study of the ethanolic extract of the aerial parts of Jatrophagossypiifolia in rats. Rev. bras. farmacogn. 2012; 22(3):663-8.doi:http://dx.doi.org/10.1590/S0102-

$695 \times 2012005000024$ $695 \times 2012000300027$

10. Mendes KDS, Silveir RCP, Galvao CM. Revisão integrativa: método de pesquisa para a incorporação de evidências na saúde e na enfermagem. Texto \& contexto enferm. 2008; I7(4):758-64.doi: http://dx.doi.org/I0.I590/SOI0407072008000400018

11. Mozzato AR, Grzybovski D. Análise de Conteúdo como Técnica de Análise de Dados Qualitativos no Campo da Administração: Potencial e Desafios. RAC. 201 I; I 5(4): 73 I47. [citado I3 Mar 2016]. Disponível em: http://www.scielo. $\mathrm{br} / \mathrm{pdf} / \mathrm{rac} / \mathrm{vI}$ 5n4/aI0vI5n4.
12. Melnyk BM, Fineout-Overholt E. Evidence based practice in nursing \&Helthcare: a guide to best practice. Philadelphia: Lippincot Williams \&Wilkins; 2005.656 p.

13. Pereira Jr LR, Andrade AP, Araújo KD, Barbosa AS, Barbosa FM. Espécies da Caatinga como Alternativa para o Desenvolvimento de Novos Fitofármacos Flor \& Amb. 20 I4; 2 I (4):509-20. doi: http://dx.doi.org/I0.I590/2 I798087.0242I 2 ISSN I4I5-0980

14. Trindade MT. Espécies úteis da família Euphorbiaceae no Brasil. Revista Cubana de Plantas Medicinales [revista en Internet]. 2015 [citado 2017 Nov II]; 19(4): I-22. Disponible en: http://www.revplantasmedicinales.sld.cu/index.php/ pla/article/view/I I 3

15. Fatokun OT, Liberty O, Esievo KB,Okhale SE, Kunle OF. Phytochemistry, Ethnomedicine and Pharmacology of Jatropha gossypiifoliaL: A Review. Arch Cur Res Intern. 2016; 5(3): I-2 I. doi: http://dx.doi.org//0.9734/ACRI/20 I6/28793

16. Mariz SR, Cerqueira GS, Araújo JGD, Duarte JC, Melo AFN, Santos HB et al. Estudo toxicológico agudo do extrato etanólico de partes aéreas de Jatropha gossypiifolia L. em ratos. Rev. bras. farmacogn.2008; 16(3):372-8.doi: http://dx.doi. org/I0.1590/S0102-695X20060003000I5.

17. Vale JR, Czeczko NG,Aquino JU, Ribas-Filho JM, Bettega L,Vasconcelos PRL et al. Estudo comparativo da cicatrização de gastrorrafias com e sem o uso do extrato de Jatropha gossypiifolia L. (pião roxo) em ratos.Acta Cir Bras. 2006; 21 (3): 40-8. doi: http://dx.doi.org/ I0.1590/S0 I02-86502006000900007

18. Santos MFS, Czeczko NG, Nassif NA, Ribas-Filho JM, Alencar BLF, Malafaia $O$ et al.Avaliação do uso do extrato bruto de Jatropha gossypiifolia L. na cicatrização de feridas cutâneas em ratos. Acta Cir Bras. 2006; 21 (3):2-7. doi:http:// dx.doi.org/ I0.1590/S0 102-86502006000900002

19. Andreollo NA, Santos EF, Araújo MR, Lopes LR. Idade dos ratos versus idade humana: qual é a relação?ABCD arq. bras. cir. dig. 2012; 25(I): 49-5I. doi: http://dx.doi.org/10.1590/ SOI02-672020I2000I000I I

20. Servin SCN, Torres OJM, Matias JEF, Agulham MA, Carvalho FA, Lemos $R$ et al.Ação do extrato de Jatropha gossypiifolia $L$. (pião roxo na cicatrização de anastomose colônica: estudo experimental em ratos. Acta Cir Bras. 2006; 21 (3):89-96. doi: http://dx.doi.org/ 10.1590/S0 102-86502006000900012

21. Maia, JMA, Czeczko NG, Ribas-Filho JM, Dietz UA, Duck $D$, Ribas CAPM et al. Estudo da cicatrização de suturas na bexiga urinária de ratos com e sem a utilização de extrato bruto de Jatropha gossypiifolia L. intraperitoneal. Acta Cir Bras. 2006; 2 I (2):26-30. doi: http://dx.doi.org/10.1590/ SOI02-86502006000800005

22. Aquino JU, Czeczko NG, Malafaia O, Dietz UA, Ribas-Filho JM, Nassif NA et al. Avaliação fitoterápica da Jatropha gossypiifolia L. na cicatrização de suturas na parede abdominal ventral de ratos. Acta Cir Bras. 2006; 2 I (2):6 I-6. doi: http:// dx.doi.org/I0.I590/S0 I02-865020060008000I0

23. Campos ACL, Borges-Branco A, Groth AK. Cicatrização 
de feridas. $A B C D$ arq. bras. cir. dig. 2007; 20(I): 5 I-58. doi: http://dx.doi.org/I0.1590/S0102-67202007000100010

24. Bhagat R, et al. Anti-inflammatory activity of Jatropha gossypifolia L. leaves in albino mice and Wistar rat. JSIR. 20I I; 70:289-92.[citado 2016 Apr 9].Disponível em: http:// nopr.niscair.res.in/bitstream/I 23456789/I I 589/I/JSIR\%20 70\%284\%29\%20289-292.pdf

25. Félix-Silva J, Giordani RB, Silva-Jr AA, Zucolotto SM, Fernandes-Pedrosa MF. Jatropha gossypiifolia L. (Euphorbiaceae): A review of traditional uses, phytochemistry, pharmacology, and toxicology of this medicinal plant. eCAM. 20I4; I4: I-
32. doi: http://dx.doi.org//0.1 | 55/2014/369204

26. Mariz SR, Araújo MST, Cerqueira GS, Araújo WC, Duarte JC, Diniz MFFM et al. Avaliação histopatológica em ratos após tratamento agudo com o extrato etanólico de partes aéreas de Jatropha gossypiifolia L. Rev. bras. Farmacogn. 2008; I8(2): 213-I6. doi: http://dx.doi.org/I0.I590/S0I02$695 \times 2008000200012$

27. Rohenkohl CC, Ana Paula Carniel AP, Colpo E. Consumo de antioxidantes durante tratamento quimioterápico. 20II; 24(2):I07-II2. doi: https://doi.org/I0.1590/S0I0267202011000200004 